\title{
Risk factors for spread of primary adult onset blepharospasm: a multicentre investigation of the Italian movement disorders study group
}

Department of

Neurological and

Psychiatric Sciences,

University of Bari,

Italy

G Defazio

P Livrea

Department of Neurology, and

Neuromed Institute,

University of Rome La

Sapienza, Italy

A Berardelli

Institute of Neurology,

University of Genova,

Italy

G Abbruzzese

CIMEDOC, University of Bari, Italy

V Coviello

G Assennato

Istituto Nazionale

Neurologico C Besta,

Milan, Italy

F Carella

Institute of

Ophtalmology,

University of Naples,

Italy

M T De Berardinis

Institute of Neurology, S. Raffaele Hospital, Milan, Italy

G Galardi

2nd Neurologic Clinic, University of Messina, Italy

P Girlanda

2nd Neurologic Clinic, University of Firenze, Italy

S Maurri

Institute of Neurology, University of Trieste, Italy

M Mucchiut

Institute of Neurology, Università Cattolica del Sacro Cuore, Rome, Italy

A Albanese

Department of Neurology, Casa Sollievo della Sofferenza, S Giovanni Rotondo, Italy

M Basciani

Giovanni Defazio, Alfredo Berardelli, Giovanni Abbruzzese, Vincenzo Coviello, Francesco Carella, Maria T De Berardinis, Giuseppe Galardi, Paolo Girlanda, Silvio Maurri, Marco Mucchiut, Alberto Albanese, Mario Basciani, Laura Bertolasi, Rocco Liguori, Nicola Tambasco, Lucio Santoro, Giorgio Assennato, Paolo Livrea

\begin{abstract}
Objectives-Little is known about factors influencing the spread of blepharospasm to other body parts. An investigation was carried out to deterrmine whether demographic features (sex, age at blepharospasm onset), putative risk, or protective factors for blepharospasm (family history of dystonia or tremor, previous head or face trauma with loss of consciousness, ocular diseases, and cigarette smoking), age related diseases (diabetes, hypertension), edentulousness, and neck or trunk trauma preceding the onset of blepharospasm could distinguish patients with blepharospasm who had spread of dystonia from those who did not.
\end{abstract}

Methods-159 outpatients presenting initially with blepharospasm were selected in 16 Italian Institutions. There were 104 patients with focal blepharospasm (mean duration of disease 5.3 (SD 1.9) years) and 55 patients in whom segmental or multifocal dystonia developed (mainly in the cranial cervical area) 1.5 (1.2) years after the onset of blepharospasm. Information was obtained from a standardised questionnaire administered by medical interviewers. A Cox regression model was used to examine the relation between the investigated variables and spread.

Results-Previous head or face trauma with loss of consciousness, age at the onset of blepharospasm, and female sex were independently associated with an increased risk of spread. A significant association was not found between spread of dystonia and previous ocular diseases, hypertension, diabetes, neck or trunk trauma, edentulousness, cigarette smoking, and family history of dystonia or tremor. An unsatisfactory study power negatively influenced the validity and accuracy of the negative findings relative to diabetes, neck or trunk trauma, and cigarette smoking.

Conclusions-The results of this exploratory study confirm that patients presenting initially with blepharospasm are most likely to experience some spread of dystonia within a few years of the onset of blepharospasm and suggest that head or face trauma with loss of consciousness preceding the onset, age at onset, and female sex may be relevant to spread. The suggested association between edentulousness and cranial cervical dystonia may be apparent because of the confounding effect of both age at onset and head or face trauma with loss of consciousness. The lack of influence of family history of dystonia on spread is consistent with previous findings indicating that the inheritance pattern is the same for focal and segmental blepharospasm.

(F Neurol Neurosurg Psychiatry 1999;67:613-619)

Keywords: blepharospasm; focal dystonia

Primary blepharospasm is an adult onset focal dystonia usually starting between the 5 th and the 7th decade and characterised by involuntary, sustained contractions of the orbicolaris oculi muscles. ${ }^{12}$ In a case-control study on possible risk factors we found that previous head or face trauma with loss of consciousness, family history of dystonia, and family history of postural tremor independently increased the risk of developing adult onset dystonia. ${ }^{3} \mathrm{~A}$ positive association was also present between local body injury and dystonia of the same body part-that is, local eye diseases and blepharospasm. ${ }^{3}$ Spontaneous remission of blepharospasm has rarely been reported, ${ }^{4}$ whereas dystonic spasms may often spread to other muscles, mainly lower face, jaw, neck, and pharyngeal and laryngeal muscles. ${ }^{125}$ In most patients dystonia spreads during the initial 5 years and in half within a year of onset of blepharospasm. ${ }^{5}$ Little is known about factors influencing the spread of blepharospasm, but the close temporal relation between onset and spread ${ }^{5}$ raises the possibility that factors preceding or accompanying the development of blepharospasm are also relevant to diffusion of dystonia to other body parts.

We investigated whether demographic features (sex, age at onset of blepharospasm), putative risk, or protective factors for blepharospasm (including family history of dystonia or tremor, previous head or face trauma, ocular diseases, and cigarette smoking), ${ }^{3}$ age related diseases (diabetes, hypertension), edentulousness, and neck or trunk trauma (thought to 
trigger dystonia in the oromandibular cervical muscles $)^{367}$ preceding the onset of blepharospasm could discriminate patients with blepharospasm who experience spread of dystonia from those who do not.

\section{Patients and methods \\ PATIENTS}

Patients were selected among consecutive outpatients with adult onset primary dystonia presenting initially with blepharospasm and attending for follow up visits at the movement disorders clinic of 14 neurological and two ophthalmological departments. Inclusion criteria were the following: a diagnosis of focal, segmental, or multifocal blepharospasm made by senior neurologists expert in movement disorders according to published standard criteria ${ }^{8}$; age at blepharospasm onset (defined as time of first symptom) $>20$ years; and duration of disease $>1$ year (to minimise possible misclassification errors in the aetiological diagnosis) and $<5$ years (to reduce recall bias). Exclusion criteria were: neurological abnormalities in addition to dystonia except tremor associated with dystonia ${ }^{9}$; a history of exposure to dopamine receptor blocking agents within 6 months before the onset of dystonia; "severe" head trauma (loss of consciousness longer than 6 hours or major surgical procedures, or both); features suggesting dopa responsive dystonia, paroxysmal dystonia, and alcohol responsive myoclonic dystonia as well as other secondary dystonias ${ }^{10}$; and features suggesting nondystonic (ophthalmological, orthopaedic, and psychogenic) causes of dystonia. ${ }^{11-13}$ The patients from the two ophtalmological centres were assessed and followed up by senior neurologists expert in movement disorders and belonging to the same University (GD and LS). One hundred and sixty five patients met the eligibility criteria, but six refused to take part in the study. One hundred and twenty two subjects were recruited from January to July 1995 in 14 centres participating in a casecontrol study on possible risk factors for primary adult onset dystonia. ${ }^{3}$ The remaining 37 patients were recruited from January to July 1996 in three additional Institutions, two neurological and one ophthalmological, according to the same criteria.

\section{DATA COLLECTION AND DEFINITION OF}

VARIABLES

Information was obtained by a standardised questionnaire administered face to face by one medical interviewer for each centre. Patients were questioned about the period preceding the onset of blepharospasm unless otherwise indicated. To maximise interpersonal and intrapersonal reliability the interviewers received training before the study. Patients were questioned about the period preceding the onset of blepharospasm unless otherwise indicated. The questionnaire collected data on age, sex, age at blepharospasm onset, medical events (including diabetes, hypertension, ocular diseases, edentulousness, non-severe head or facial trauma with loss of consciousness, and neck or trunk trauma without injury to the head or loss of consciousness), age at menopause, cigarette smoking, and family history (first degree relatives only) of postural nonparkinsonian and non-alcohol related tremor and dystonia. Information concerning medical history was supported by medical records or detailed reports of specific treatments. To elicit information on edentulousness, patients with segmental or multifocal dystonia were questioned about the period preceding both the onset of blepharospasm and spread of dystonia, those with focal blepharospasm about the period preceding the interview. Dental status was also subsequently monitored until the end of the study. Questions on cigarette smoking referred to the year preceding the onset of blepharospasm. Because the results of an earlier study suggested a protective role of current cigarette smoking on the development of dystonia, ${ }^{3}$ subjects were classified as nonsmokers (including never and past smokers) or current smokers. First degree relatives were considered to be affected by dystonia or postural tremor only if at least one other family member provided adequate information (probable diagnosis) or one of the investigators personally observed the secondary cases (definite diagnosis). The time interval between risk factor and onset of blepharospasm symptoms was recorded. In addition, the questionnaire focused on the spatial distribution of dystonia. For each involved body part the date of initial involvement (determined to within 1 year) was recorded based on patient's recollection, records from other doctors when available, and our own finding.

After inclusion in the study and completing the questionnaire patients were periodically seen until 31 December 1997. At each follow up visit, the extent of dystonia was assessed by the senior neurologist. In addition, the date of involvement of new body parts, dental status, and current medication were recorded.

\section{DATA ANALYSIS}

To examine the relation between the investigated variables and spread of dystonia the Cox proportional hazards survival analysis ${ }^{14}$ was performed by a standard statistical package (EGRET). In survival analysis interest centres on a point event occurring after a length of time, often called "the failure time". The failure time in this study was the time from the onset of blepharospasm to the initial spread. In the analysis of the time to spread, patients with focal blepharospasm at last examination represented censored data. Most exposure variables were represented in the model by a single indicator variable, ${ }^{1}$ if the subject was exposed; 0 , if not); for sex, a value of 1 was assigned to women. The quantitative variable age at blepharospasm onset was first analysed as a continuous variable, then categorised according to demographic and clinical consideration (see results). Records containing missing information were excluded from the analysis. To assess the influence of each variable on the outcome, the hazard ratio and two sided 95\% confidence intervals (95\% CIs) were calculated. The statistical significance of the hazard 
Table 1 Demographic and clinical features of the studied patients and results of questionnaire administration (number of records containing missing information is in parenthesis)

\begin{tabular}{llll}
\hline & All Patients & $\begin{array}{l}\text { Focal } \\
\text { blepharospasm }\end{array}$ & $\begin{array}{l}\text { Blepharospasm as part } \\
\text { of segmental or } \\
\text { multifocal dystonia }\end{array}$ \\
\hline Variables & 159 & 104 & 55 \\
Number & $41 / 118$ & $33 / 71$ & $9 / 46$ \\
Men/women & $58.5(9.7)$ & $57.1(9.1)$ & $61.2(7.1)$ \\
Mean duration (y) of illness (SD) & $5.2(1.8)$ & $5.2(1.7)$ & $5.3(1.9)$ \\
Ocular diseases & 48 & 31 & 17 \\
Hypertension & 34 & 20 & 14 \\
Diabetes & 9 & 6 & 3 \\
Head or face trauma with loss of & $11(2)$ & $3(1)$ & $8(1)$ \\
$\quad$ consciousness & $9(2)$ & $7(1)$ & $2(1)$ \\
$\begin{array}{l}\text { Neck or trunk trauma } \\
\text { Menopausal state preceding }\end{array} \quad$ & & 39 \\
$\quad$ blepharospasm onset & 94 & 55 & 7 \\
$\begin{array}{l}\text { Edentulousness } \\
\text { Cigarette smoking (current smokers) }\end{array}$ & 11 & 5 & 7 \\
Family history of dystonia & 23 & 16 & 4 \\
Family history of tremor & 12 & 8 & 2 \\
\hline
\end{tabular}

ratio was estimated by the likelihood ratio statistic, and $\mathrm{p}$ values $<0.05$ were considered to be significant. To check for type II error, the statistical power relative to each variable was assessed by the equation reported by Parmar and Marchin. ${ }^{15}$ For multivariate analysis we used the model building strategy proposed by Hosmer and Lemeshaw. ${ }^{16}$ Because multivariate models are unsuitable for samples having less than 10 times as many events of interest as candidate regressors, we used the 0.25 level at the univariate test as a screening criterion for selecting candidate variables. ${ }^{16}$ After fitting the model containing all the selected variables, we deleted the unimportant variables and fitted a new model. The importance of variables was assessed by examining the significance of each $\mathrm{p}$ value and comparing the estimated hazard ratios from the new and the old models. This process of deleting, refitting, and verifying continued until it yielded a model containing the essential variables only (main effects model). Thereafter, we consider the need for including biologically plausible multiplicative interaction terms among the variables. Finally, the goodness of fit (that is, the appropriatedness of the Cox model in describing the observed data) relative to the variables significantly associated with the outcome in the multivariate model was assessed. To this aim, the proportional hazards assumption was graphically checked by plotting the log-logistic model. ${ }^{15}$

\section{Results}

At the time of interview 108 patients had isolated blepharospasm and 51 had blepharospasm onset segmental or multifocal dystonia. The 159 patients had an overall mean disease duration of 3.1 (SD 1.3) years. They were followed up for 1.5 to 3 years. Only four patients spread during the follow up period. At the last examination, therefore, there were 104 cases with isolated blepharospasm and 55 with blepharospasm onset segmental or multifocal dystonia: their overall mean duration of disease was 5.2 (SD 1.8) years. Brain CT, or MRI, or both showed normality in 102 patients $(64 \%)$, in the remaining 57 patients $(36 \%)$ no imaging studies were available. The 57 did not show a significantly higher frequency of risk factors for brain damage (including diabetes, hypertension, and cigarette smoking) than patients with normal head imaging findings. Brain CT, MRI, or both were available for all patients who had a history of head trauma. Patients with and without head imaging studies had comparable mean (SD) disease duration (5.3 (1.9) years $v$ 5.2 (1.4) years).

Demographic and clinical features of the patients studied and answers to the questionnaire are listed in table 1 . Among antecedent events there were ocular diseases (blepharitis keratoconjunctivitis, glaucoma, cataract, and retinopathy) 1 to 5 years before the onset of blepharospasm, head or face trauma with loss of consciousness (lasting some minutes to 2 hours) 1 to 6 years before the onset of blepharospasm, and neck or trunk trauma 1 to 7 years before the onset of blepharospasm. Menopausal state preceded the onset of blepharospasm in about $80 \%$ of female patients. In the year before onset, 23 patients smoked and 11 were edentulous. Dental status was also monitored during the follow up period, but none of the patients reported losing their teeth after the onset of blepharospasm. Twelve patients reported a family history of dystonia (most affected relatives had the same type of dystonia as the cases) and 15 patients a history of postural tremor. Three patients reported family history of both dystonia and tremor (in different family members). Eight patients had relatives with a definite diagnosis of dystonia and 10 of postural tremor. Patients from neurological and ophtalmological centres, as well as the 122 patients recruited during 1995 and the 37 recruited during 1996 did not differ in regard to demographic and clinical features (not shown).

Stratifying patients by duration of disease at interview showed no increase in the relative frequency of risk factors across strata as the duration of disease increased (not shown).

After the onset of blepharospasm, most patients were treated with botulinum toxin and less than $8 \%$ of patients experienced other medications, including anticholinergic drugs and benzodiazepines. These patients were equally distributed among patients with and without spread.

Among the 55 patients in whom dystonia spread, jaw or lower face muscles were involved in $43(78 \%)$, cervical muscles in $15(27 \%)$,

Table 2 Results of univariate Cox regression analysis

\begin{tabular}{|c|c|c|c|}
\hline & $\begin{array}{l}\text { Hazard } \\
\text { ratio }\end{array}$ & $95 \% C I$ & $p$ Value \\
\hline \multicolumn{4}{|l|}{ Variables } \\
\hline Age at blepharospasm onset & 1.06 & 1.02 to 1.1 & 0.004 \\
\hline Sex (female) & 3.8 & 1.3 to 11.3 & 0.018 \\
\hline Ocular diseases & 1.12 & 0.6 to 1.9 & 0.7 \\
\hline Hypertension & 1.3 & 0.8 to 2 & 0.4 \\
\hline Diabetes & 0.96 & 0.4 to 2.5 & 1 \\
\hline \multicolumn{4}{|l|}{ Head or face trauma with } \\
\hline loss of consciousness & 4.8 & 1.9 to 11.5 & $<0.001$ \\
\hline Neck or trunk trauma & 0.43 & 0.1 to 2.3 & 0.25 \\
\hline Edentulousness & 2.69 & 1.2 to 5.9 & 0.015 \\
\hline $\begin{array}{l}\text { Cigarette smoking (current } \\
\text { smokers) }\end{array}$ & 1.11 & 0.5 to 2.3 & 0.78 \\
\hline Family history of dystonia & 0.81 & 0.3 to 2.3 & 0.69 \\
\hline Family history of tremor & 0.28 & 0.1 to 1.2 & 0.08 \\
\hline
\end{tabular}


Table 3 Results of multivariate analysis

\begin{tabular}{lllr}
\hline & $\begin{array}{l}\text { Hazard } \\
\text { ratio }\end{array}$ & $95 \%$ CI & p Value \\
\hline Variables & & & \\
$\quad$ Head/face trauma with loss & 5.7 & $2.2-14.4$ & $<0.001$ \\
$\quad \begin{array}{l}\text { of consciousness } \\
\text { Age at blepharospasm onset }\end{array}$ & 1.06 & $1.02-1.1$ & 0.006 \\
Sex (female gender) & 3.9 & $1.3-11.8$ & 0.017 \\
\hline
\end{tabular}

laryngeal muscles in four (7\%), and arm muscles in three $(5 \%)$. In 13 patients dystonia spread at two extrapalpebral sites. Overall, patients had some spread of dystonia within 5 years after blepharospasm began: About half of the patients $(55 \%)$ spread within a year of blepharospasm onset, $22 \%$ within 2 years, $16 \%$ within 3 years, $7 \%$ within 4 years, and $2 \%$ within 5 years. The mean duration of disease before spreading to one body part was 1.5 (SD 1.2) years, and (SD 1.7) 3.1 years before spreading to two parts. The average (SD) durations of disease before spreading to the jaw or lower face (1.3 (0.9) years), larynx (2.1 (0.6) years), neck (2.2 (1.5) years) and upper limbs (4.3 (0.5) years) seemed to be consistent with a somatotopic progression of dystonia (one way analysis of variance (ANOVA): degrees of freedom=63, $F=9.6$, p $<0.0001$; Newman-Keuls post hoc test: jaw or lower face different from neck and upper extremities, $\mathrm{p}<0.05$; neck different from jaw or lower face and upper extremities, $p<0.005$; upper extremities different from each other group, $\mathrm{p}<0.05)$. For the 104 patients without spread, the mean duration of disease ( SD) was significantly much longer than the mean time required for initial spread of symptoms (5.3 (1.7) years; range 4 to 8 years $v 1.5$ (1.2 years); range 0.1 to 5 years, $\mathrm{p}<0.01$ ).

When analysing initial spread by the Cox regression model, variables which failed to reach significance on univariate analysis included previous ocular disease, hypertension, diabetes, neck or trunk trauma, cigarette smoking, and family history of dystonia or tremor (table 2). Conversely, previous head or face trauma with loss of consciousness, age at blepharospasm onset, sex, and edentulousness were significantly associated with the outcome (table 2). The study had an estimated $<80 \%$ chance of detecting three times modification in the risk of spread with $\alpha=0.05$ (two sided) for prior diabetes, neck or trunk trauma, and cigarette smoking. The other variables calculated yielded a study power $>90 \%$.

Table 4 Distribution of previous head or face trauma with loss of consciousness, female sex, and age at blepharospasm onset among patients with focal blepharospasm, spread at one body part, and spread at two body parts

\begin{tabular}{|c|c|c|c|}
\hline Variables & $\begin{array}{l}\text { Patients with focal } \\
\text { blepharospasm } \\
(n=104)\end{array}$ & $\begin{array}{l}\text { Patients with } \\
\text { spread at one body } \\
\text { part }(n=42)\end{array}$ & $\begin{array}{l}\text { Patients with spread } \\
\text { at two body parts } \\
(n=13)\end{array}$ \\
\hline $\begin{array}{l}\text { Head or face trauma with loss of } \\
\text { consiousness }\end{array}$ & $3(3 \%)$ & $5(12 \%)$ & $3(23 \%)$ \\
\hline Female sex & $71(68 \%)$ & $36(86 \%)$ & $12(92 \%)$ \\
\hline $\begin{array}{l}\text { Age at blepharospasm onset } \\
(\text { mean (SD)) }\end{array}$ & $53(9)$ & $57(14)$ & $64(3)$ \\
\hline
\end{tabular}

$\chi^{2}(2 \times 3)$ : Head or face trauma with loss of consiousness, $\mathrm{p}=0.009$; Female sex, $\mathrm{p}=0.09$. One way ANOVA: age at blepharospasm onset, $F=7.7$, p $<0.0001$; Newman-Keuls post hoc test: each group different from the others, $\mathrm{p}<0.05$.
There was an inverse relation between age at onset of blepharospasm and time to initial spread $(r=-0.28, t=2.1, \mathrm{p}=0.04)$. To assess further the relation between risk of spread and age at onset of blepharospasm, the continuous variable was categorised into three separate categories. The boundaries for these categories were derived in such a way as to make divisions at numerically simple values, to ensure about $33 \%$ of patients in each category, and to make clinical sense. Taking age at blepharospasm onset $<55$ years as reference yielded hazard ratios of 2.04 (95\% CI 0.94 to 4.44 ) for age at onset of blepharospasm between 55 and 63 years and of 3.43 (95\% CI 1.6 to 7.4 ) for age at onset of blepharospasm $>63$ years (score test for trend of rates: $\left.\chi^{2} 16.01 ; \mathrm{p}<0.001\right)$. Thus, patients with older age at onset of blepharospasm were most likely to experience spread.

The main effects model (table 3 ) included previous head or face trauma with loss of consciousness, age at onset of blepharospasm (continuous variable), and sex as independent risk factors. Multivariate analysis showed that the significant effect of edentulousness on univariate analysis was distorted because it was mixed with the effect of both age at blepharospasm onset and prior head or face trauma with loss of consciousness (confounding effect). Yet the same results were obtained when more homogeneous case groups-that is, the 104 patients with focal blepharospasm and the 43 patients with orofacial dystonia-were analysed to verify whether edentulousness related more strongly to orofacial than to other dystonias. Interaction terms among age at blepharospasm onset, sex and prior head or face trauma with loss of consciousness never attained $\mathrm{p}$ values $<0.4$ (possibly because not enough patients showed the combined risk factors needed for calculating interaction). Variables included in the final model satisfied the proportional hazards assumption.

Because of the age and sex related difference in the risk of spread we examined whether there was some relation between the menopausal state and spread. Mean (SD) age at menopause did not significantly differ between female patients with and without spread (48.2 (5.6) v 50.3 (4.6), Mann Whitney $U$ test, $\mathrm{p}=0.11)$. Menopausal state preceded the onset of blepharospasm in $85 \%$ of patients who spread and in $78 \%$ of patients who did not $(\mathrm{p}=0.51)$.

Due to the size of the sample we could not use the Cox model to separately examine spread at more than one body part. Thus we calculated the distribution of the variables included in the final model in the 104 patients with focal blepharospasm, in the 42 with spread at one body part, and in the 13 with spread at two body parts. It must be stressed that the number of patients remaining with spread at one body part at the end of the study period was unlikely to change significantly because their mean duration of disease (SD) was much longer than the mean time required for spread at two parts $(5.4(1.9)$ v 3.1 (1.7) years; $p<0.001)$. The frequency of previous head or face trauma with loss of consciousness 
(but not of female sex) and the average age at blepharospasm onset across strata increased with the extent of spread (table 4).

\section{Discussion}

We found that in about $35 \%$ of patients dystonia developed elsewhere within 5 years of the onset of blepharospasm. Oromandibular and cervical muscles were the most frequent sites of progression. The percentage of patients remaining with focal blepharospasm at the end of the study period was unlikely to change significantly because the mean duration of disease was much longer than the mean time required for initial spread. Overall, the figures for rate and extent of spread were in agreement with previous reports that found the initial 5 years to be crucial for spread of blepharospasm, ${ }^{5}$ and a greater and earlier involvement of oromandibular muscles compared with cervical and limb muscles. ${ }^{12}$

Of the previous clinical studies on blepharospasm, none specifically focused on prognostic indicators of progression. Because of the close temporal relation between blepharospasm onset and spread (present study and ${ }^{5}$ ) we conducted an exploratory study designed to evaluate whether demographic features, putative risk, or protective factors for blepharospasm, ${ }^{3}$ age related diseases (diabetes, hypertension), edentulousness, and neck or trunk trauma ${ }^{367}$ preceding or accompanying the onset of blepharospasm influenced spread. Due to the few patients with spread at two extrapalpebral sites, Cox analysis focused on the time it took patients to involve one site. Multivariate analysis indicated that previous head or face trauma with loss of consciousness, age at blepharospasm onset, and female sex independently increased the risk of spread in patients presenting initially with blepharospasm. In addition, we found that the frequency of head and face trauma with loss of consciousness but not of female sex, and the average age at blepharospasm onset increased across the subgroups of patients with focal blepharospasm, spread at one body part, and spread at two body parts as the extent of spread increased.

Theoretically, errors in classifying subjects as having focal or segmental blepharospasm may have caused distortion. In this study, the diagnosis of focal or segmental blepharospasm was made by senior neurologists expert in movement disorders. Previously, we checked the ability of six of them to correctly identify patients with focal or segmental blepharospasm. ${ }^{17}$ By means of $\mathrm{K}$ statistics, a substantial between observer agreement $(k=0.63, \mathrm{p}<0.001)$ was found. ${ }^{17}$ This result suggests that errors in the diagnosis of focal or segmental blepharospasm are unlikely among neurologists expert in movement disorders.

Because the study was not population based, we cannot rule out a bias in patient selection. To correct for this we included patients from a large number of clinics in various areas of Italy and recruited $96 \%$ of the consecutive outpatients who met eligibility criteria during the study period. Although $40 \%$ of patients did not have brain CT or MRI, eligibility criteria made contamination from secondary dystonia unlikely. ${ }^{18}$ In addition, we found no significant difference in the frequency of risk factors potentially responsible for brain damage in patients with and those without imaging studies.

Although the sample was limited to primary adult onset cases in whom blepharospasm had been the initial presenting symptom, our patient population had demographic and clinical features similar to those of previous clinical series. ${ }^{12}$ In most patients, the course of dystonia was reconstructed retrospectively. Because patients tend to recall disability whereas mild symptoms may be forgotten or unrecognised, the date of initial involvement of each body part was based not only on the patient's recollection but also on medical records and our own findings. Although we did not base the study on newly diagnosed patients, the relatively short average duration of disease at study inclusion reduced, but did not eliminate, the potential for recall bias. Using prevalent patients may be a potential source of survival bias, but the low incidence of blepharospasm in the general population ${ }^{19}$ made it not feasible to use incident cases and to perform a prospective study. None the less, survival bias seems unlikely because stratifying the cases by disease duration showed no increase in the relative frequency of risk factors across strata as disease duration lengthened.

We failed to find a significant association between spread of dystonia and previous ocular diseases, hypertension, diabetes, neck or trunk trauma, edentulousness, cigarette smoking, and family history of dystonia or tremor. An unsatisfactory study power negatively influenced the validity and accuracy of the negative findings relative to previous diabetes, neck or trunk trauma, and cigarette smoking. The inclusion of a larger number of patients could clarify these issues. The reason why we found a lower rate of familial occurrence of dystonia than did previous series ${ }^{20}{ }^{21}$ could be that we identified affected relatives from the case patients' answers to the questionnaire rather than by clinical examination of all at risk relatives. Overall, we may have identified only a proportion of familial cases, thus making an information bias in the direction of the null value likely. Alhough the hazard ratio calculated for family history of postural tremor did not reach significance, the $95 \%$ CI suggests that the variable may be associated with a decreased risk of spread. Besides an information bias, the potential misclassification error in distinguishing the aetiological and clinical type of tremor should be considered because postural tremor may be a feature of many neurological disorders and data on family history were, at least partly, self reported. Postural tremor may also be an initial manifestation of dystonia. ${ }^{9}$ Hence we cannot be sure that some relatives reported as having tremor alone actually had dystonia. The possible relation between family history of postural tremor and spread needs, therefore, to be further evaluated. 
Because of a satisfactory study power, the negative findings relative to previous ocular disease, hypertension, and edentulism were more robust and probably valid. Differential recall of ocular diseases between cases with focal and segmental or multifocal blepharospasm is unlikely. Data concerning edentate patients deserve further comment. Previous anecdotal observation suggested that edentulousness could predispose to orofacial, neck, and trunk dyskinesia by disrupting dental proprioception. ${ }^{67}$ Because the onset of dystonia may be prolonged and difficult to detect, ${ }^{22}$ others suspected that involuntary orofacial movements possibly contribute to dental problems. ${ }^{2}$ In our sample, all patients reported edentulousness before the onset of blepharospasm. However, the significant univariate estimate relative to this variable was distorted (even when the subgroup of patients with orofacial dystonia was separately examined) by a confounding effect of both age at blepharospasm onset and head or face trauma, thus suggesting that the association between edentulousness and spread may be apparent. Other potential risk factors for orofacial dystonia, including dental procedures, oral surgery, and bruxism, were not investigated because their retrospective recognition may be limited by patient recall or lack of objective assessment.

Results indicated prior head or face trauma with loss of consciousness as a significant risk factor for spread of dystonia in patients presenting initially with blepharospasm. This association raises the question of whether patients with segmental or multifocal blepharospasm may have better recall for head or face trauma than patients with focal blepharospasm. Yet events inducing a loss of consciousness should be recalled equally well by both categories of patients. In all cases more than a year elapsed between trauma, blepharospasm onset, and diffusion of dystonia. These long time intervals do not necessarily argue against a causal relation because delayed onset dystonia has been reported after injuries. ${ }^{23}{ }^{24}$ Overall, we conclude that head or face trauma with loss of consciousness preceding blepharospasm may be relevant to spread. The positive association between spread of dystonia (mainly in the cranial cervical area) and head or face trauma with loss of consciousness in patients with neither clinical nor radiographic evidence of brain damage may be consistent with the suggested role of peripheral trauma in inducing topographically related dystonia. ${ }^{232526}$ The area involved makes it difficult or impossible; however, to exclude direct discrete damage to brain regions putatively involved in the pathophysiology of dystonia.

The finding that patients with older age at the onset of blepharospasm are most likely to experience spread is by striking contrast with spread of symptoms in childhood and juvenile onset dystonia. In this setting, children with younger age at onset have a higher risk of developing widespread dystonia and experience more rapid spread of dystonia than older children. ${ }^{5}$ None the less, supporting the validity of our findings, patients presenting initially with adult onset torticollis and writer's cramp have a younger age at onset than patients presenting initially with blepharospasm and are also less prone to the development of segmental or multifocal dystonia. ${ }^{27}$ In addition, we found that the higher the age at the onset of blepharospasm, the shorter the time to spread and the more the number of body parts involved. The age related difference in the risk of and time to spread may just be a function of aging. Yet multivariate analysis excluded a potentially confounding role of age related diseases including hypertension and diabetes. Older patients with blepharospasm may be more likely than younger patients with blepharospasm to experience conditions, possibly triggering dystonia in predisposed subjects. These may include trauma, edentulousness, and neuroleptic drug intake. Alhough we did not specifically investigate the matter, none of our patients reported loss of teeth or neuroleptic drug intake after the onset of blepharospasm. Alternatively, the age related difference in the risk of spread may reflect age related changes in D2 mediated inhibition of striatopallidal inhibitory neurons of the indirect pathway which has recently been implicated in the pathophysiology of dystonia. ${ }^{29}$

Multivariate analysis indicated a positive association between female sex and initial spread, but there was no difference in the fequency of women between subgroups of patients with different extent of spread. The much greater number of women affected by segmental or multifocal dystonia is possibly due to a selection bias, but the model used takes into account the female preponderance when analysing hazard ratios for this factor. It must be stressed that many large studies on cranial-cervical dystonia showed a similar preponderance of females to males. ${ }^{12}{ }^{30}$ It has been suggested that women may be more at risk for development and spread of dystonia because of specific estrogen receptors within the CNS which could influence involuntary motor function. ${ }^{31}$ Due to the age related differences in the risk of spread we looked for a relation between menopause and spread. However, age at menopause was not significantly different between female patients with and without spread, and menopausal state preceding the onset of blepharospasm was not more frequent in women who spread than in those who did not.

In conclusion, this study confirms that patients presenting initially with blepharospasm are most likely to experience some spread of dystonia within a few years of the onset of blepharospasm, and suggests that age at onset of blepharospasm, female sex, and factors preceding the development of blepharospasm (head or face trauma with loss of consciousness) may be relevant to spread. Interestingly, the hazard ratios and their $95 \%$ CIs indicate that previous head or face trauma with loss of consciousness may have a role greater than the other factors. The suggested association between edentulousness and cranial cervical dystonia may result from confounding by other variables. Because history alone is not a reliable 
method to identify family members affected by dystonia, the lack of influence of family history of dystonia on spread agree with findings indicating that the inheritance pattern is the same for focal and segmental blepharospasm. ${ }^{20}{ }^{21}$

\section{Appendix}

List of participants

R De Salvia, M Giorelli, Department of of Neurological and Psychiatric Sciences, University of Bari, Italy; L Vacca, Department of Neurology, and Neuromed Institute, University of Rome "La Sapienza", Italy; R Marchese, Institute of Neurology, University of Genova, Italy; L Capus, Institute of Neurology, University of Trieste, Italy; V Fetoni, Istituto Nazionale Neurologico C Besta, Milan, Italy; P Calace, G Cortese, Institute of Ophtalmology, University of Naples, Italy; L Maderna, Institute of Neurology, S Raffaele Hospital, Milan, Italy;

A Trusso, 2nd Neurologic Clinic, University of Messina, Italy; S Brogelli, 2nd Neurologic Clinic, University of Firenze, Italy; A R Bentivoglio, the Institute of Neurology, "Università Cattolica del Sacro Cuore", Rome, Italy; P Simone, Department of Neurology, "Casa Sollievo della Sofferenza", S Giovanni Rotondo, Italy; C Cordivari, Institute of Neurology, University of Bologna, Italy; A Rossi, F Lalli, Institute of Neurology, University of Perugia, Italy; C Crisci, F Crispi, 2nd Neurologic Clinic, University of Naples, Italy.

1 Grandas F, Elston J, Quinn N, et al. Blepharospasm: a review of 264 patients. $\mathcal{F}$ Neurol Neurosurg Psychiatry 1988;51:76772

2 Jankovic J, Ford J. Blepharospasm and orofacial-cervica dystonia:clinical and pharmacological findings in 100 patients. Ann Neurol 1988;13:402-11

3 Defazio G, Berardelli A, Abbruzzese G, et al. Possible risk factors for primary adult-onset dystonia: a case-control investigation by the Italian Movement Disorders Study Group. F Neurol Neurosurg Psychiatry 1998;64:25-32.

4 Defazio G, Lamberti P, Lepore V, et al. Facial dystonia: clinical features, prognosis and pharmacology in 31 patients. Ital f Neurol Sci 1989;10:553-60.

5 Greene P, Kang UJ, Fahn S. Spread of symptoms in idiopathic torsion dystonia. Mov Disord 1995;10:143-52.

6 Sutcher HD, Underwood RB, Beatty RA, et al. Orofacial Sutcher HD, Underwood RB, Beatty RA, et al. Orofacial
dyskinesia, a dental dimension. $7 A M A 1971 ; 216.1459-63$

7 Sandyk R, Kay SR. Edentulousness and neurolepticSandyk R, Kay SR. Edentulousness and neurolepticinduced

8 Fahn S. Concept and classification of dystonia. In: Fahn S, Marsden CD, Calne DB, eds. Advances in neurology. Vol 50 New York, Raven Press, 1988:1-8

9 Jedynak CP, Bonnett AM, Agid Y. Tremor and idiopathic dystonia. Mov Disord 1991;6:230-6.
10 Calne DB, Lang AE. Secondary dystonia. In: Fahn S, Marsden CD, Calne DB, eds. Advances in neurology. Vol 50 , New York, Raven Press, 1988:9-33.

11 Salorio PD, Conte RQ. Ophthalmologic causes of blepharospasm. In: Jankovic J, Tolosa E, eds. Advances in neurology. Vol 49. New York, Raven Press, 1988:91-102.

12 Suchowersky O, Calne DB. Non-dystonic causes of torticollis. In: Fahn S, Marsden CD, Calne DB, eds. Dystonia 2. Advances in neurology. Vol 50. New York, Raven Press, 1988:501-8.

13 Fahn S, Williams DT. Psychogenic dystonia. In: In: Fahn S, Marsden CD, Calne DB, eds. Dystonia 2. Advances in neurology. Vol 50. New York, Raven Press, 1988:431-55.

14 Cox DR. Regression models and life tables. Fournal of the Royal Statistical Society 1972;34:187-220.

15 Parmar KB, Machin D. Survival analysis: a practical approach. New York: John Wiley, 1995.

16 Hosmer DW, Lemeshaw S. Applied logistic regression. New York, John Wiley, 1989.

17 Defazio G, Lepore V, Abbruzzese G, et al. Reliability among neurologists in the severity assessment of blepharospasm and oromandibular dystonia: a multicenter study. Mov Disord 1994;9:616-21.

18 Marsden CD. Investigation on dystonia. In: Fahn S, Marsden CD, Calne DB, eds. Dystonia 2. Advances in neu rology. Vol 50. New York: Raven Press, 1988:35-43.

19 Nutt JG, Muenter MD, Aronson A, et al. Epidemiology of focal and generalized and dystonia in Rochester, Minnesota. Mov Disord 1988;3:188-94

20 Waddy HM, Fletcher MD, Harding AE, et al. A genetic study of idiopathic focal dystonias. Ann Neurol 1991;29. $320-4$.

21 Defazio G, Livrea P, Guanti G, et al. Genetic contribution to idiopathic adult-onset blepharospasm and cranial-cervical dystonia. Eur Neurol 1993;33:345-50.

22 Micheli S, Fernandez-Pardal M, Quesada P, et al. Variable onset of adult inherited focal dystonia: a problem for genetic study. Mov Disord 1994;9:64-8.

23 Scott B, Jankovic J. Delayed-onset progressive movement disorders after static brain lesions. Neurology 1996;46:6874.

24 Lee MS, Rinne JO, Ceballos-Baumann A, et al. Dystonia after head trauma. Neurology 1994;44:1374-8.

25 Jankovic J. Post-traumatic movement disorders: central and peripheral mechanisms. Neurology 1994;44:2006-14.

26 Fletcher NA, Harding AE, Marsden CD. The relationship between trauma and idiopathic torsion dystonia. $\mathcal{F}$ Neurol Neurosurg Psychiatry 1991;54:713-7.

27 Jahanshahi M, Marion M-H, Marsden CD. Natural history of adult-onset idiopathic torticollis. Arch Neurol 1990;7: 548-52.

28 Jankovic J, Leder S, Warner D, et al. Cervical dystonia: clinical findings and associated movement disorders. Neurology 1991;41:1088-91.

29 Perlmutter JS, Tempel LW, Black KJ, et al. MPTP induces dystonia and parkinsonism. Clues to the pathophysiology of dystonia. Neurology 1997;49:1432-8.

30 Soland VL, Kailash P, Bhatia P, et al. Sex prevalence of focal dystonias. F Neurol Neurosurg Psychiatry 1996;60:204-5.

31 Duane DD. Spasmodic torticollis. In: Fahn S, Marsden CD, Calne DB, eds. Dystonia 2. Advances in neurology. Vol 50. New York: Raven Press, 1988:525-35. 